\title{
Audit of knee injuries seen in a sports medicine clinic
}

\author{
G. J. Packer FRCSEd, G. R. McLatchie FRCS*, W. Bowden FRCS and \\ C. M. E. Lennox FRCSEd(Orth) \\ Departments of General and Orthopaedic Surgery, Hartlepool General Hospital, Hartlepool, \\ Cleveland TS24 9AH, UK and *National Sports Medicine Institute, London, UK
}

\begin{abstract}
In order to assess the management of knee injuries in a sports medicine clinic, an audit was performed of all new patients who attended the clinic over a 12-month period. Of the 167 new patients seen, $76(46 \%)$ had sustained knee injuries. Of these, $43(57 \%)$ were treated in the sports medicine clinic and $33(43 \%)$ were referred for arthroscopic assessment. The maximum waiting time from the time of referral was 3 weeks, with $85 \%$ of patients seen within 1 week and $92 \%$ within 2 weeks. Arthroscopy was performed on $28(85 \%)$ of the 33 patients referred, and the positive correlation between the sports clinic diagnosis and the arthroscopic diagnosis was $64 \%$. Of the 33 patients referred for arthroscopy, $28(85 \%)$ had sustained acute knee injuries while five (15\%) had been treated at other hospitals before referral to the sports medicine clinic. A National Health Service sports medicine clinic is an effective means of treating knee injuries, provided that access to arthroscopy is readily available.
\end{abstract}

Keywords: Sports medicine clinic, knee injuries, audit

The knee joint is commonly injured as a result of sporting activity and the importance of early and appropriate treatment of these injuries has been emphasized previously ${ }^{1}$.

A National Health Service sports medicine clinic was initiated at Hartlepool General Hospital in 1989, run weekly by a consultant general surgeon and a consultant in accident and emergency medicine, both of whom have commitments to sports medicine. Referrals are accepted from general practitioners, hospital doctors and club physiotherapists.

An audit was performed in order to assess the effect of a sports medicine clinic on the treatment of knee injuries.

\section{Patients and methods}

A retrospective analysis of the clinical notes of all new patients referred to the sports medicine clinic over a 12-month period (from 1 January 1991 to 31 December 1991) was performed in order to establish the number of patients with knee injuries and the treatment they received.

\section{Results}

Over the period of the audit a total of 167 new patients was seen, of which $76(46 \%)$ had sustained knee injuries. Of the patients with knee injuries, 43 $(57 \%)$ were treated in the sports medicine clinic and $33(43 \%)$ required referral to the orthopaedic service for consideration for arthroscopic diagnosis or treatment.

The clinical diagnoses of all the patients with knee injuries are shown in Table 1.

Of the 33 patients referred for arthroscopy, 28 $(85 \%)$ underwent the procedure, of the remaining five patients, two failed to attend for their orthopaedic appointment and three were treated by non-operative means. The clinical diagnosis of the sports medicine clinic was confirmed by arthroscopy in $64 \%$ of patients.

Acute knee injuries accounted for 28 of the patients referred for arthroscopy, the remaining five patients $(15 \%)$ had already undergone treatment for their knee injury at another hospital before referral to the sports medicine clinic. Each of these five patients underwent arthroscopy and all five had significant

Table 1. Clinical diagnoses of patients with knee injuries

\begin{tabular}{lc}
\hline Diagnosis & Number \\
\hline Patients treated in the sports clinic & \\
Meniscal injury & 6 \\
Medial ligament injury & 6 \\
Chondromalacia patellae & 6 \\
Anterior knee pain & 4 \\
No definite diagnosis & 4 \\
Osteoarthritis & 3 \\
lleotibial friction band & 3 \\
Hamstring injury & 3 \\
Osgood-Schlatter disease & 3 \\
Patellar tendinitis & 2 \\
Prepatellar bursitis & 1 \\
Anterior cruciate injury & 1 \\
Arthropathy & 1 \\
Total & 43 \\
Patients referred for arthroscopy & \\
Medial meniscus injury & \\
Lateral meniscus injury & 20 \\
Anterior cruciate injury & 3 \\
Chondromalacia patellae & 7 \\
Total & 3 \\
\hline
\end{tabular}

Address for correspondence: Mr G. J. Pácker, Department of Orthopaedic Surgery, Royal Victoria Infirmary, Queen Victoria Road, Newcastle upon Tyne NE1 4LF, UK

C) 1993 Butterworth-Heinemann Ltd 0306-3674/93/020113-02 
pathology (a meniscal tear and/or rupture of the anterior cruciate ligament).

The maximum waiting time from referral to an appointment in the sports medicine clinic was 3 weeks, although $85 \%$ of patients were seen within 1 week and $92 \%$ within 2 weeks.

\section{Discussion}

Over the period of the audit, almost half of the new patients referred to the sports medicine clinic had sustained knee injuries. It seems likely, therefore, that in the future sports medicine clinics will fulfil an increasingly important role in the treatment of acute knee injuries. This role has previously been undertaken by accident and emergency departments, although previous studies ${ }^{2,3}$ have emphasized the difficulties that casualty officers face in dealing with these patients because of their inexperience in the diagnosis and treatment of acute knee injuries.

All of the patients with knee injuries were seen within 3 weeks and more than $80 \%$ within 1 week. This waiting time contrasts significantly with the long local waiting list for orthopaedic outpatients (currently 3 months for a 'soon' appointment). Of the patients with knee injuries, $43 \%$ were referred to the orthopaedic department in order to assess their need for arthroscopy. This assessment was undertaken by a local orthopaedic consultant; patients being seen in outpatients the week after referral from the sports medicine clinic. As a result of the audit it became clear that this arrangement was unsatisfactory, and so an acute knee clinic is now run by the orthopaedic department concurrently with the sports medicine clinic. This knee clinic also accepts acute referrals from the accident department and has open access to arthroscopy. The majority of patients who required arthroscopy had sustained acute knee injuries, although five $(15 \%)$ were referred because of continuing symptoms and had previously been treated elsewhere. Each of these patients had a significant knee lesion at arthroscopy, emphasizing the importance of the clinic in acting as a safety net for patients with continuing problems.

This audit demonstrates that a National Health Service sports medicine clinic is an effective means of treating knee injuries. The high yield of arthroscopic findings in those referred $(64 \%)$ suggests that special interest clinics of this nature, run by senior staff, not only allow early recognition of potentially serious knee injuries but also act as a safety net for those with continuing problems.

\section{References}

1 Baugher WH, White GM. Primary evaluation and treatment of knee injuries. Orthop Clin North Am 1985; 16: 315-17.

2 O'Bierne J, O'Dwyer T, O'Rourke JS, Quinlan W. The diagnosis of knee injuries in casualty - a prospective study. Injury 1983; 15: 232-5.

3 Packer GJ, Gayner AD, Craxford AD, Caullay JM, Goring CC. Effect of an algorithm on the treatment of knee injuries. Injury 1992; 23: 270-2. 